\title{
Débat sur la Politique agricole commune après
}

\section{3}

\section{(2) OpenEdition \\ Journals}

Édition électronique

URL : http://journals.openedition.org/economierurale/3917

DOI : $10.4000 /$ economierurale.3917

ISSN : 2105-2581

Éditeur

Société Française d'Économie Rurale (SFER)

Édition imprimée

Date de publication : 15 mars 2013

Pagination : 99-105

ISSN : 0013-0559

\section{Référence électronique}

"Débat sur la Politique agricole commune après 2013 », Économie rurale [En ligne], 334 | mars-avril 2013, mis en ligne le 15 mars 2015, consulté le 21 avril 2019. URL : http://journals.openedition.org/ economierurale/3917 ; DOI : 10.4000/economierurale.3917 


\section{DÉBAT}

\section{Débat sur la Politique agricole commune après 2013}

La rubrique "Débat " d'Économie rurale change de forme. Elle s'appuie désormais sur des interventions dans le cadre des Séminaires de politiques agricoles (SPA) organisés par la Société française d'économie rurale. Sur un sujet d'actualité, deux points de vue courts ${ }^{1}$ sont demandés aux intéressés. L'introduction de ce débat a été rédigée par Aurélie Trouvé, responsable de cette rubrique. (NDLR.)

a Politique agricole commune (PAC) peut connaître une réforme importante après 2013. La Commission européenne a présenté en novembre 2011 ses propositions législatives ${ }^{2}$. Elles comprennent notamment :

- l'harmonisation progressive des montants d'aides directes entre agriculteurs, entre régions et entre États membres ;

- le greening, c'est-à-dire des conditions environnementales appliquées à $30 \%$ des montants d'aides directes (maintien des pâturages permanents, diversification des cultures, maintien d'une surface d'intérêt écologique) ;

- le reversement de l'enveloppe nationale allouée aux aides directes (i) vers des exploitations en zones soumises à des contraintes naturelles (jusqu'à $5 \%$ de l'enveloppe, mesure facultative), (ii) vers les jeunes agriculteurs (jusqu'à $2 \%$ de l'enveloppe, mesure obligatoire), (iii) vers les petites exploitations (qui recevront si elles choisissent ce traitement entre 500 et 1000 euros par an), (iv) vers le $2^{\text {d }}$ pilier (jusqu'à $10 \%$ de l'enveloppe, mesure facultative) ;

- la possibilité de re-coupler les aides directes jusqu'à 5 ou $10 \%$ de l'enveloppe nationale ;

1. De moins de 9000 signes chacun.

2. http://ec.europa.eu/agriculture/cap-post-2013/ legal-proposals/index_fr.htm.
- le plafonnement des aides à 300000 euros par an, avec des réductions d'aides à partir de 150000 euros ;

- l'octroi des aides uniquement aux agriculteurs considérés comme actifs ;

- une clause de sauvegarde, c'est-à-dire des mesures d'urgence en termes d'intervention publique et d'aide au stockage privé lors de perturbations générales du marché ;

- la suppression des quotas pour le sucre ;

- la reconnaissance des organisations de producteurs et interprofessionnelles dans tous les secteurs, avec un financement transféré vers le $2^{\mathrm{d}}$ pilier (de même que le financement des outils comme les assurances privées et les fonds de mutualisation) ;

- dans le $2^{\mathrm{d}}$ pilier, la suppression des 4 axes et la création de 6 priorités.

Beaucoup de critiques se sont exprimées en France, pour des raisons parfois différentes. Ainsi, le greening a été jugé trop ambitieux par la profession agricole majoritaire et bien trop timoré par des organisations environnementales, la Confédération paysanne, les collectivités régionales... Les mêmes clivages sont apparus sur l'homogénéisation des montants d'aides directes. Mais beaucoup se sont retrouvés pour critiquer la poursuite de la dérégulation des marchés, notamment des volumes de production, et l'inefficacité relative des alternatives proposées (assurances privées, 
renforcement des organisations de producteurs et des interprofessions... $)^{3}$.

Ces débats nationaux nourrissent les débats entre États membres et au sein du Parlement européen : des négociations s'y déroulent, avec l'objectif d'un double accord d'ici 2014 sur la PAC et le financement de l'UE. Mais ces propositions peuvent-elles vraiment apporter une nouvelle légitimité sociale et environnementale à la PAC ? C'est la question qui est posée aux deux contributeurs de cette rubrique : Tomás García Azcárate ${ }^{4}$ et Régis Hochart ${ }^{5}$, lors des séminaires du 8 novembre 2011 et du 14 février 2012. Le premier se concentre sur les possibles avancées, à partir de deux sujets fortement débattus dans le cadre de cette réforme, le verdissement des aides directes et l'équilibre de la chaîne alimentaire (notamment, la coordination et le partage de la valeur ajoutée entre acteurs de la filière). Le second analyse de façon plus critique la proposition législative en soulignant les écarts avec ses préconisations.

\section{Point de vue de Tomás García Azcárate}

Je voudrais réfléchir sur deux sujets qu'il me semble important d'avoir en tête à l'heure de l'analyse de la proposition de réforme de la PAC : le verdissement des aides et l'équilibre de la chaîne alimentaire.

3. Trouvé A., Lataste F. G. (2011). Synthesis and assessment of the public debate on the reform of the CAP after 2013 - the case of France. Rapport pour un projet de recherche, IFLS-Goethe Université, http://www2.dijon.inra.fr/esr/pagesperso/trouve/ French\%20Report \%20Final\%20Version.pdf.

4. Conseiller pour l'analyse économique des marchés agricoles à la DG Agri, Maître de conférences de l'Institut d'études européennes de l'Université Libre de Bruxelles, membre de l'Académie d'agriculture de France, tomas.garcia-azcarate@ec europa.eu, Page web: www.tomasgarciaazcarate.eu. 5. Régis Hochart, membre du Conseil économique, social et environnementale, au titre de la Confédération Paysanne, rapporteur de l'avis « $L a$ future PAC après 2013 », CESE 25 mai 2011.

\section{Le verdissement des aides}

En ce qui concerne le verdissement, j'aimerais aborder le lien entre la proposition de réforme et les défis de l'alimentation des habitants du monde, à l'horizon 2050 par exemple.

Pour augmenter de $60 \%$ (nouveau calcul de la FAO) la production agricole entre 2005 et 2050, il faut une augmentation de la production utilisée de l'ordre de $1 \%$ par an, alors que depuis 1961 on observe une croissance de plus de $2 \%$. De plus, on peut réduire les pertes avant récolte, durant le stockage et le transport, dans la filière et les ménages ; la FAO estime aussi qu'une politique d'égalité de traitement entre les hommes et les femmes augmenterait la production mondiale entre 10 et $20 \%$.

Disons le clairement, ce n'est pas aux pays développés en général et à l'Europe, pour ce qui nous concerne, d'alimenter les pauvres du monde qui sont dans leur majorité des paysans ou des habitants du monde rural, c'est au monde de s'alimenter.

Toutefois, le maintien de prix mondiaux fermes à l'avenir pour les céréales conduit à mon avis à un vrai risque de «céréalisation » de l'agriculture européenne. Même avec la hausse des coûts de production - induite par la hausse du prix de l'énergie et donc des carburants - des fertilisants et autres intrants, la production céréalière reste rentable, avec un niveau de risque limité et une présence physique moindre sur l'exploitation que dans l'élevage, par exemple. Ainsi, le rapport de septembre 2011 de l'Institut de l'élevage sur «L'élevage allaitant français à l'horizon 2015 et perspectives 2035 » signale bien l'attrait pour les grandes cultures dans les zones mixtes.

Cette céréalisation de l'agriculture européenne, résultat de l'addition logique de décisions microéconomiques et individuelles de chaque entrepreneur en lien 
direct avec le marché, représenterait une mutation considérable et consoliderait l'éloignement entre les demandes de la société (et sa disponibilité pour financer l'agriculture européenne) et le monde agricole. Cela sonnerait le glas du soutien public à l'agriculture et impliquerait une restructuration massive. D'ailleurs, parmi les opposants au verdissement, se trouvent tous ceux qui veulent en finir avec, ou au moins réduire la voilure de la PAC en ayant un œil aussi bien sur les discussions budgétaires de la prochaine période 20142020 qu'en préparant le terrain pour la PAC post-2020. Quelle aubaine ce serait pour eux de priver la PAC d'une grande partie de sa légitimité !

La Commission propose donc le verdissement des aides de la PAC sur le premier pilier, avec trois obligations qui sont trois remparts contre la céréalisation : le maintien des prairies permanentes, la diversité des productions et la préservation de réservoirs écologiques et des paysages. Et ceci doit se faire sur l'ensemble du territoire, et non pas sur les $20 \%$ de Surface Agricole Utile couverts par les mesures agroenvironnementales, ainsi qu'avec les fonds du premier pilier, c'est-à-dire sans cofinancement national ou régional obligatoire, toujours aléatoire dans le contexte budgétaire actuel.

\section{L'équilibre de la chaîne alimentaire}

Dans le contexte actuel, les propositions doivent être extrêmement mesurées dès qu'elles ont un impact budgétaire. Les propositions qui n'ont pas ou peu de coûts budgétaires doivent être examinées avec attention.

Il s'agit, par exemple, de la politique de qualité et de promotion des produits alimentaires, des programmes pour les fruits et légumes ou le lait à l'école et de l'aide alimentaire aux plus démunis. Mais il s'agit aussi de l'équilibre de la chaîne alimentaire pour éviter des spirales destructrices de valeur, au détriment de tous les acteurs et, en premier lieu, du monde agricole et des petites et moyennes industries.

Le sujet n'est pas nouveau. Déjà en 1969 on pouvait lire dans le Mémorandum Mansholt que « l'amélioration de la structure des marchés et des conditions de commercialisation est indispensable si l'on veut que les producteurs tirent pleinement parti des possibilités offertes par le marché unique ». On y parlait, entre autres, de « concentration quantitative et d'adaptation qualitative de l'offre », d' « accroissement de la transparence du marché », d' " organiser la diffusion permanente de l'ensemble des offres et des demandes présentes sur le marché, ainsi que de l'état des stocks », de la nécessaire « discipline des producteurs », de groupements de producteurs capables « au début de chaque campagne, en fonction des perspectives annuelles des marchés, d'étudier les conditions et les disciplines de mise en marché » et de bien d'autres choses.

Dans sa communication de 1990 sur les organisations interprofessionnelles, la Commission précisait que « l'objectif de rendre plus flexibles les mécanismes de soutien aux marchés n'est pas de remplacer l'ordre par l'anarchie mais de stimuler l'apparition de nouvelles structures dans lesquelles les producteurs et leurs organisations doivent jouer un rôle plus actif ».

D'abord, il convient de signaler que, même sans aucun changement, des possibilités importantes existent déjà. La Direction générale de la concurrence de la Commission européenne a élaboré un rapport intitulé : How EU competition policy helps dairy farmers in Europe, où on souligne les marges actuellement existantes sans aucun changement législatif. On n'y trouve pas de réponses à tous les problèmes mais il fourmille d'éléments utiles pour trouver des solutions dans un nombre non négligeable de cas. 
En outre, des exceptions aux normes européennes de concurrence sont possibles, pourvu que soient respectés les principes de transparence, de proportionnalité et d'efficacité. Autrement dit, les mesures qui pourraient être couvertes par ces exceptions doivent explicitement être prévues dans l'OCM unique, limitées à ce qui est strictement nécessaire pour l'accomplissement des objectifs de la mesure et efficacement contribuer à l'accomplissement de chacun des objectifs assignés par le Traité à la politique agricole, y compris assurer des prix raisonnables aux consommateurs.

C'est la raison pour laquelle la proposition de la Commission comporte un important chapitre sur les organisations des producteurs, leurs associations et les interprofessions. Il s'agit de consolider l'expérience acquise dans des secteurs comme le lait, les fruits et légumes ou le vin en sachant qu'il n'y a pas de réponse générale et généralisable pour tous les produits agricoles et tous les États membres.

En cohérence d'ailleurs avec ce qui est proposé dans le cadre de l'OCM unique, une priorité claire est accordée aux activités dites de «coopération» entre les acteurs de la filière dans le cadre du développement rural.

Avec le Traité de Lisbonne, l'approbation de la future PAC fera l'objet d'une codécision entre le Parlement européen et le Conseil. La proposition de la Commission reflète un équilibre entre la protection des consommateurs et celle des acteurs de la filière, entre la distribution et l'industrie, entre les industriels, et en premier lieu les petites et moyennes entreprises, et le monde agricole, entre l'objectif de compétitivité et celui d'équité, enfin entre la légitime défense du revenu des producteurs et la nécessaire lutte contre l'inflation.

Mais rien ne garantit que l'équilibre proposé par la Commission soit celui du Conseil et du Parlement européen. Nous n'avons pas le droit à l'échec. La réussite passe par une réflexion pondérée qui prenne en compte l'ensemble des acteurs de la filière, des producteurs aux consommateurs. Pour cela, il faut que les mondes agricole et de la concurrence se comprennent, se parlent et se dessinent une image, comme dirait le Petit Prince.

\section{Point de vue de Régis Hochart}

Déterminer le sens d'un projet politique ne peut se faire qu'à condition de faire le constat de la situation, puis au vu de ce constat et à la lumière des enjeux, d'énoncer des orientations. C'est alors seulement que l'on peut estimer la pertinence d'un projet politique.

\section{Le constat}

Aujourd'hui, un milliard d'habitants sont sous-alimentés. Ceux qui à travers le monde consacrent plus de la moitié de leur budget familial aux produits alimentaires se retrouvent dans l'impossibilité d'y accéder si les prix doublent. La volatilité des prix agricoles et alimentaires est un fléau, d'autant plus qu'il enlève au paysan toute visibilité pour faire des choix de production.

L'évolution de l'agriculture des six dernières décennies a été fondée sur quatre piliers :

- Les ressources fossiles, le phosphore et la potasse qui ont permis des gains de productivité mais sont en voie d'épuisement et de renchérissement. Certaines sont de fortes productrices de gaz à effets de serre.

- Les produits phytosanitaires qui, s'ils ont apporté temporairement des réponses à certains problèmes, ont dégradé tous les processus biologiques des sols et des animaux d'élevage, tout en faisant courir 
des risques sur la santé des utilisateurs et des consommateurs.

- La mécanisation qui a amélioré les conditions de travail des agriculteurs, mais est aujourd'hui destructrice d'emploi, dans un monde où le chômage et la précarité croissent vertigineusement.

- Les processus de sélection qui ont permis une très forte augmentation de la productivité, mais ont réduit le patrimoine génétique disponible, et aliéné les paysans.

Le système d'échanges entre les États mis en place à la suite des accords du Gatt puis de l'OMC a mis en concurrence des systèmes agricoles où la productivité par travailleur n'est pas du tout la même, annihilant les efforts de développement agricole de nombreuses régions.

\section{Les orientations : ce qu'il convient de faire}

Stabiliser les prix des denrées agricoles est un enjeu économique et social essentiel. Il importe de mettre en œuvre tous les outils qui peuvent y contribuer : des mécanismes d'intervention doivent accompagner la mise en place de stocks stratégiques de produits végétaux. L'UE seule ne peut y suffire, mais elle doit y contribuer. La souveraineté alimentaire doit être instaurée et des écluses tarifaires entre les différentes régions du monde doivent être acceptées dans un principe de réciprocité. Les marchés à terme doivent être supprimés ou à tout le moins l'accès à ces marchés doit être interdit aux opérateurs financiers spéculateurs. La gestion des volumes produits est un facteur essentiel de stabilisation des prix. À ce titre, la production d'agrocarburants ne peut se poursuivre ainsi car elle amplifie la volatilité des prix des productions végétales. Elle doit être limitée à une transformation d'éventuels excédents de production.
Les systèmes de production développés depuis 1960 sont aujourd'hui obsolètes. Nous sommes au bout de cette approche « chimique » de l'agriculture. Pour être durable, l'agriculture devra s'appuyer sur la biologie - des sols, des écosystèmes. La recherche, le partage des savoirs, le développement devront s'orienter vers l'agroécologie. Plus que le rendement d'une production par hectare ou par animal, c'est la productivité globale au niveau d'un territoire qui devra être la référence, prenant en compte le maintien de la fertilité des sols et de la biodiversité, la qualité des eaux, la limitation de la production de GES... Pour y parvenir, une déspécialisation des territoires est nécessaire, induisant la diversification des productions, la synergie entre élevage et productions végétales et la généralisation de la culture des légumineuses dans les rotations.

Parce que le chômage devient un problème majeur, il est aberrant de vouloir poursuivre une mécanisation outrancière. La mécanisation doit servir à l'amélioration des conditions de travail des paysans et non plus à la substitution au travail. Développer une agriculture et un secteur de la transformation riches en main-d'œuvre doit être un objectif politique.

Sept milliards de personnes doivent avoir accès à l'alimentation, il est donc nécessaire que tous les hectares agricoles soient productifs et que l'UE améliore son autonomie alimentaire. C'est devenu un enjeu stratégique : les populations des autres régions du monde vont-elles durablement accepter que leurs terres servent à nous nourrir ? Rappelons que contrairement à une idée durablement entretenue par le concept erroné de « vocation exportatrice de l'Europe », nous sommes importateurs nets de denrées agricoles, pour un équivalent de 29 millions d'hectares, non comptés les aliments tropicaux, pour nous nourrir. C'est par la mise en place d'une agriculture relocalisée que nous trouverons les réponses. 


\section{Une absence inquiétante de vision prospective}

La communication de la Commission européenne du 18 novembre 2010 montrait avec éclat que ni la stabilisation des prix agricoles ni la gestion des volumes produits ne faisait partie de ses objectifs, seul restant valable pour elle le rôle du marché. À l'inverse, la volonté de soutenir l'emploi, de concilier environnement et production, de mettre en place un soutien aux légumineuses et protéagineux, de soutenir les petites fermes, dont on sait qu'elles sont capables d'être tout aussi productives que les autres, laissaient augurer une prise de conscience de la Commission européenne d'une partie des enjeux.

La publication des propositions législatives de la Commission le 12 octobre 2011 a clarifié la situation : il s'agissait seulement de bonnes intentions. Et la future PAC n'est en rien à la hauteur des enjeux. Elle poursuit dans la voie de l'abandon des mesures de régulation et de la gestion des volumes produits : elle ne remet pas en cause les paiements découplés ni la surface comme référence des soutiens, elle propose une dégressivité et un plafonnement très faibles des aides, elle n'envisage pas de paiements contracycliques, qui consisteraient à soutenir les producteurs en fonction des revenus attendus de l'année. La proposition de la PAC après 2013 est en l'état actuel dans la continuité des réformes précédentes.

Il est du devoir des pouvoirs publics français de chercher dans les 18 mois prochains des alliances avec d'autres États membres pour infléchir la ligne actuelle de la Commission européenne. Il est du devoir du Parlement européen de proposer une évolution profonde de ces propositions : un renforcement considérable de la dégressivité et du plafonnement des aides, l'aide aux petites fermes complémentaire des autres aides du $1^{\text {er }}$ pilier (et non exclusive, tel que proposé actuellement par la Commission), la prise en compte de tous les emplois et pas seulement dans les très grandes exploitations, un projet de développement rural qui renforce l'aide au développement des territoires ruraux (actuel axe 3 du second pilier), le maintien des outils de régulation des volumes produits, un niveau d'aides couplées plus important...

Il est du devoir de la gauche paysanne française, associations, syndicats, chercheurs et penseurs, riche de réflexions et d'actions sur les territoires, bien vivante même si elle est moins visible, de faire pression sur les institutions et les pouvoirs publics. Son ancrage dans les mouvements européens renforce sa légitimité pour, contrairement à la commission, proposer une vision à moyen et long terme.

Il faudra également utiliser toutes les latitudes que ce projet va offrir à chaque État membre dans la répartition des soutiens pour donner un minimum de sens à une politique agricole et territorialisée.

La Commission propose des mesures dans le cadre du $1^{\text {er }}$ pilier :

- réserver jusqu'à $2 \%$ de l'enveloppe nationale pour augmenter de $25 \%$ le soutien de base aux nouveaux installés. C'est une mesure qu'il convient d'appuyer ;

- coupler les aides pour des productions et/ou des territoires spécifiques. Pour la France, nous pouvons monter jusqu'à $11 \%$ de l'enveloppe nationale du $1^{\text {er }}$ pilier. Nous pouvons réserver $5 \%$ de l'enveloppe nationale pour mieux doter les productions dans les territoires à fragilités naturelles et $10 \%$ pour soutenir les petites fermes.

Nous devons maximiser l'utilisation de toutes ces possibilités et rechercher tous les moyens qui permettraient un soutien plus important sur les premiers hectares, comme c'est déjà le cas pour la prime à l'herbe. Le paiement de base en sera réduit d'autant. Le paiement vert qui représente 
$30 \%$ de l'enveloppe nationale serait, en l'état actuel, et contrairement à ce qu'essaient de nous faire croire certains, accessible à tous sans gros efforts. À ce niveaulà, ce doit être considéré plutôt comme une harmonisation européenne à des actions que la France a déjà globalement mises en œuvre.

Dans le $2^{\mathrm{d}}$ pilier, les mesures agroenvironnementales, conçues comme des actions collectives sur des micro-territoires, le soutien aux nouveaux installés et le développement des territoires ruraux peuvent être la colonne vertébrale du futur développement rural. Quant au soutien proposé aux assurances, il n'a rien à faire dans le $2^{\mathrm{d}}$ pilier, comme le propose la Commission européenne!

Dans la mesure où tous les hectares français deviendraient éligibles, et où les pouvoirs publics utiliseraient tous les leviers ci-dessus, le paiement de base s'abaisserait à un niveau de l'ordre de 140 à $145 €$ par ha, une fois réalisée la convergence - qui doit être rapide -, auquel il faut rajouter le paiement vert qui pourrait se situer à $100 €$ par hectare. Restent ensuite les autres opportunités offertes par la Commission dans le $1^{\text {er }}$ et le $2^{\mathrm{d}}$ pilier (ce dernier devant être régionalisé, à l'exception de l'ICHN, mesure de solidarité par essence) : ces paiements additionnels peuvent rendre les soutiens un peu plus intelligents, devant permettre le maintien des productions dont nous avons besoin et un revenu pour tous les paysans. Ce sera une question de volonté politique des élus français. Ce sera la possibilité d'éviter le pire et de commencer à construire l'agriculture et l'alimentation de demain, en attendant une réforme en profondeur qui ne devra pas se laisser désirer trop longtemps. 